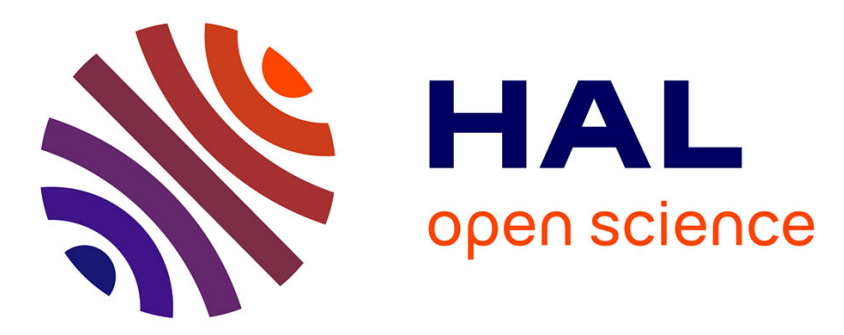

\title{
Les couches minces semiconductrices
}

M. Rodot, C. Paparoditis

\section{To cite this version:}

M. Rodot, C. Paparoditis. Les couches minces semiconductrices. Revue de Physique Appliquée, 1966, 1 (3), pp.160-160. 10.1051/rphysap:0196600103016000 . jpa-00242707

\section{HAL Id: jpa-00242707 https://hal.science/jpa-00242707}

Submitted on 1 Jan 1966

HAL is a multi-disciplinary open access archive for the deposit and dissemination of scientific research documents, whether they are published or not. The documents may come from teaching and research institutions in France or abroad, or from public or private research centers.
L'archive ouverte pluridisciplinaire HAL, est destinée au dépôt et à la diffusion de documents scientifiques de niveau recherche, publiés ou non, émanant des établissements d'enseignement et de recherche français ou étrangers, des laboratoires publics ou privés. 


\title{
LES COUCHES MINCES SEMICONDUCTRICES
}

\author{
M. RODOT et C. PAPARODITIS \\ Laboratoire de Magnétisme et de Physique du Solide, C. N. R. S., Bellevue, France.
}

Résumé. - On analyse d'abord les deux facteurs fondamentaux dont dépend la qualité des couches minces semiconductrices : l'exactitude de leur composition stoechiométrique et la perfection de leur structure. Ces facteurs sont plus ou moins bien contrôlés suivant la nature du support et la méthode de préparation. Cette dernière peut mettre en œuvre différentes forces élémentaires, pour entraîner les particules à déposer sur le support : gradient thermique (évaporation de type classique), différence de potentiel chimique (évaporation-diffusion en régime isotherme [1]) ou combinaison des deux (transport en phase vapeur). On rappelle les caractéristiques thermodynamiques du phénomène de sublimation d'une part, et d'autre part le rôle des défauts ponctuels ou quasi-ponctuels sur les caractéristiques des composés semiconducteurs [2].

La mobilité électronique est un indice sensible de la perfection structurelle des couches. Entre les couches microcristallines et les couches épitaxiées, il existe une structure à cristallisation dirigée, conduisant à une mobilité élevée, mais pour certains matériaux seulement [3]. Parmi ceux-ci se trouvent ceux qu'utilise la technique des photopiles et en particulier CdTe et les tellurures de cuivre, qui sont étudiés de façon plus approfondie à titre d'exemple. On décrit en particulier comment peuvent être obtenues des couches de CdTe quasi-monocristallines, de mobilité 200 à $400 \mathrm{~cm}^{2} / \mathrm{V}$.s et de résistivité $10^{3}$ à $10^{5} \Omega \mathrm{cm}$ [4], et des couches de $\mathrm{Cu}_{1,85} \mathrm{Te}$, qui allient une faible résistivité à une forte transparence [3].

\section{BIBLIOGRAPHIE}

[1] Cohen-Solal (G.), Marfaing (Y.) et Bailly (F.), Rev. Phys. Appl., 1966, 11,1.

[2] Rodot (H.), J. Phys. Chem. Solids, 1964, 25, 85 ; C. R. Acad. Sc., 1964, 268, 6386 ;

Rodot (H.), Triboulet (R.), Hruby (A.), Proc. Int. Symp. on Lattice Defects, Tokyo 1966, (à paraître).
[3] Paparoditis (C.), Sella (C.), Darimagna (D.) (at Bernard (J.), Commun. Coll. Internat. Plis;. Couches Minces, Clausthal-Göttingen, 1965, p. 732 .

[4] Paparoditis (C.) et Rodot (M.), Commun. Coll. Internat. Phys. Couches Minces, ClausthilGöttingen, 1965, p. 666. 